\title{
High-throughput genotyping of advanced congenic lines by high resolution melting analysis for identification of Bbaa2, a QTL controlling Lyme arthritis
}

Kenneth K.C. Bramwell ${ }^{1}$, Ying Ma ${ }^{1}$, John H. Weis ${ }^{1}$, Cory Teuscher ${ }^{2}$ and Janis J. Weis ${ }^{1}$

${ }^{I}$ Department of Pathology, University of Utah, Salt Lake City, UT, USA, and ${ }^{2}$ Department of Medicine, University of Vermont, Burlington, VT, USA

BioTechniques 52:183-190 (March 2012) doi 10.2144/000113838

Keywords: High Resolution Melting Analysis; Congenic Mice; Genotyping; Lyme Arthritis; Backcrossing; Fine Mapping

Supplementary material for this article is available at www.BioTechniques.com/article/113838

Congenic mapping is a powerful strategy to identify genomic loci regulating quantitative traits. Generating congenic lines is an iterative process of refinement that is both time and resource intensive. Here we report an alternative to traditional microsatellite marker analysis or costly high-density oligonucleotide single nucleotide polymorphism (SNP) arrays for congenic genotyping. The identification of inherited SNP variability in congenic lines using high resolution melting analysis (HRMA) represents a novel application of the method. The blocked probe HRMA approach offers a scalable, low cost, closed-tube system that benefits from rapid turnaround times, and unequivocal interpretation. The markedly higher prevalence of SNPs relative to microsatellites in the genome allows much greater flexibility for the identification of new genotyping landmarks as congenic intervals are refined. We have adopted this approach in our development of B6.C3-Bbaa2 congenic lines for the identification of loci regulating murine Lyme arthritis severity. As a result, we have been able to fully genotype individuals prior to weaning age, and expand our number of breeding cages without increasing our colony budget. Thus far, 26 SNP markers have been successfully mapped to the Bbaa2 locus. This has facilitated the identification of 20 novel B6.C3-Bbaa 2 congenic lines spanning the original interval.

The central dogma of molecular biology is that heritable differences in DNA lead to changes in mRNA transcripts, proteins, and ultimately to observable variations in phenotypes such as disease susceptibility (1). Inbred mouse strains provide an invaluable resource of fixed genomic variability to scientifically investigate this genetic component of disease susceptibility. The ability to generate intercross populations between susceptible and resistant inbred mouse strains, combined with powerful statistical analysis, allows regulatory loci of quantitative traits to be mapped to regions of individual chromosomes. Once identified, the breeding of congenic mouse lines allows individual regulatory loci to be isolated in the context of a controlled genetic background, and then to be narrowed to finer resolution in an unbiased manner through additional backcrossing to parental lines. The genetic resolution is achieveable through such breeding strategies is limited by the number and position of experimentally discernable genetic landmarks across the region of interest. Microsatellite markers have long been a key resource for genetic mapping due to their prevalence and ease of analysis by PCR.

The Bbaa2 murine Lyme arthritis susceptibility locus was identified in multiple independent intercrosses between resistant and susceptible inbred strains (2-3). Roper et al. used composite interval mapping (CIM) on reciprocal backcross populations between $\mathrm{C} 3 \mathrm{H} /$ $\mathrm{HeN}(\mathrm{C} 3 \mathrm{H})$ and either C57BL/6 (B6) or Balb/c to identify complex regulation by up to four putative QTL within Bbaa2Bbaa3, with a maximum
LOD score of 10.2. Reciprocal interval specific congenic lines (ISCLs) for the Bbaa2Bbaa 3 region on chromosome 5 developed on both the $\mathrm{B} 6$ and $\mathrm{C} 3 \mathrm{H}$ backgrounds each retained a significant portion of the disease susceptibility phenotype conferred by the parental line (4). However, the full B6.C3Bbaa2Bbaa3 congenic interval spanned 72.29 - 141.16 Mbp and included more than 870 genes and ESTs, making candidate gene selection impractical.

Efforts have been made to further refine regulatory loci within Bbaa2 to a resolution of $1 \mathrm{Mb}$ or narrower through additional ISCL backcrossing. However, given the putative complexity of the Bbaa2 locus, the availability of microsatellite markers across the interval became a limiting factor, prompting the evaluation of alternative genotyping strat- 
egies. At present, the dbSNP database contains more than 8,000 single nucleotide polymorphisms (SNPs) which differ between $\mathrm{B} 6$ and $\mathrm{C} 3 \mathrm{H}$ inbred lines across the Bbaa2 interval (5). Although the $\mathrm{C} 3 \mathrm{H} / \mathrm{HeN}$ mouse strain used to
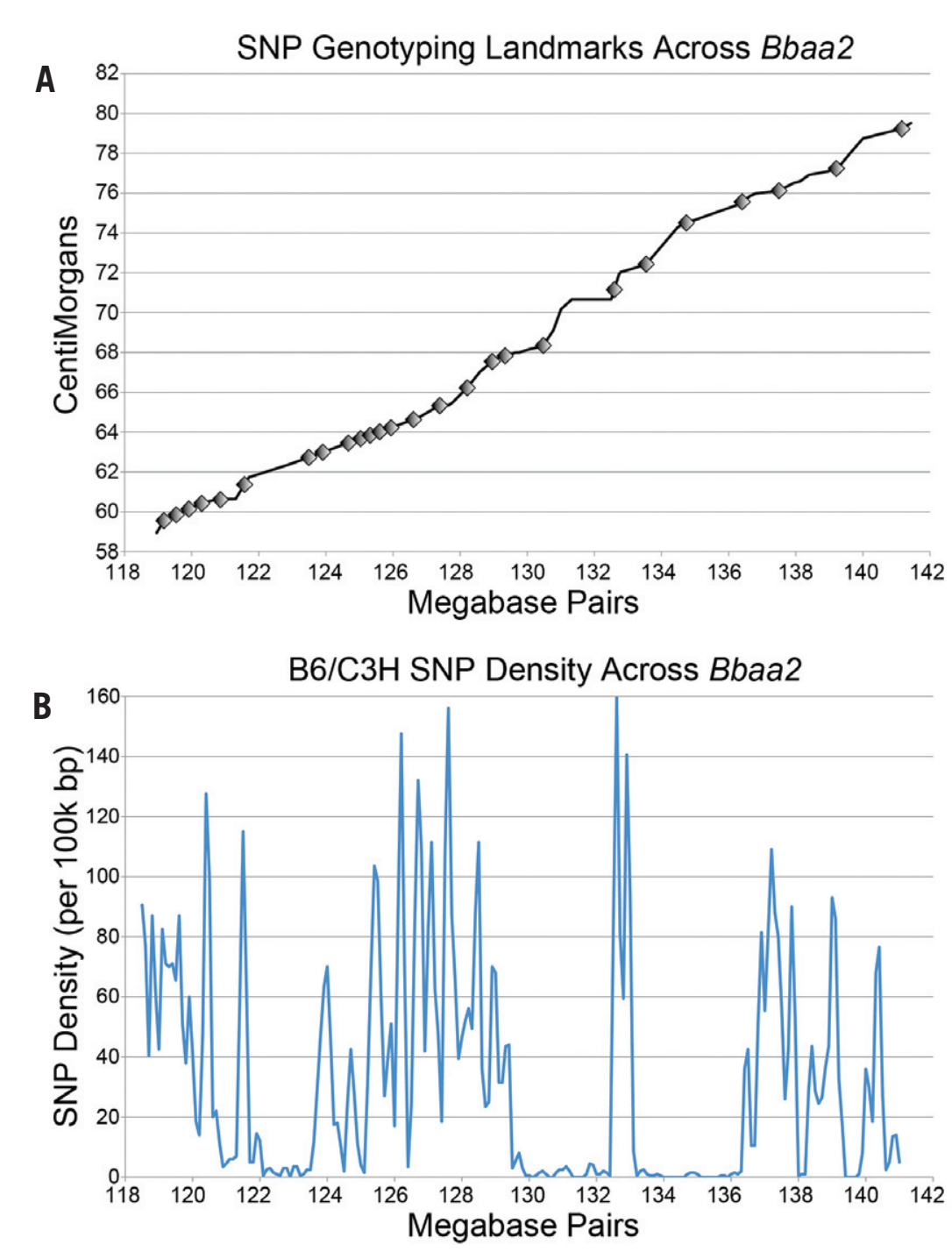

Figure 1. Distribution of SNP Genotyping Assays and SNP Density Across Bbaa2. (A) Microsatellites from the MGD database (http://www.informatics.jax.org/) with assigned cM values were plotted against their basepair position to obtain a linear plot of $\mathrm{CM}$ versus genomic location (Black Line). Markers indicate the positions of SNP Genotyping Assays developed across the interval. (B) SNP Density across Bbaa2 was calculated from all dissimilar B6/C3H SNPs present in the MGD database.
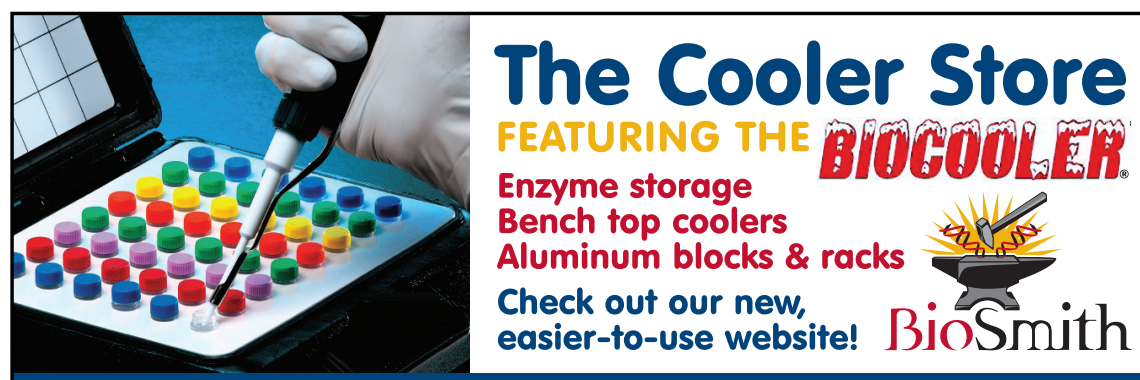

BioSmith.com

858.693.3476 create our congenic lines is not one of the eighteen currently included in the Sanger SNP re-sequencing project (6), data for the closely related $\mathrm{C} 3 \mathrm{H} / \mathrm{HeJ}$ strain is available. The Sanger database contains over 19,000 high quality SNPs which differ between $\mathrm{C} 3 \mathrm{H} / \mathrm{HeJ}$ and $\mathrm{C} 57 \mathrm{BL} / 6 \mathrm{NJ}$ SNPs in the Bbaa2 interval. A SNP genotyping strategy thus offered the potential to dramatically improve genetic resolution by increasing the number of discernable landmarks available. Several previously described SNP genotyping methodologies were evaluated: amplification refractory mutation system (ARMS)-PCR (7), small amplicon high resolution melting analysis (HRMA) (8), and blocked probe HRMA (9). We found that both methods of HRMA were useful for the genotyping of inherited intervals in congenic animals, with blocked probe HRMA being especially practical. Recent studies have emphasized the application of HRMA analysis for clinical diagnostics, but its application toward precise characterization of congenic mice has not previously been described.

\section{Materials and methods}

All mice used in this study were maintained in a pathogen free facility, and cared for in accordance with protocols approved by the University of Utah Institutional Animal Care and Use Committee (IACUC).

Genomic DNA was prepared from 2-3 mm tail clips from 14 to 17 day old mice by incubating in $600 \mu \mathrm{L}$ of $50 \mathrm{mM}$ $\mathrm{NaOH}$ at $95 \mathrm{C}$ for one hour, following by neutralization with $50 \mu \mathrm{L}$ of $1 \mathrm{M}$ Tris- $\mathrm{HCl} \mathrm{pH}$ 8. Samples were then centrifuged at $6000 \times \mathrm{g}$ for $6 \mathrm{~min}$ in a tabletop centrifuge and transferred to a clean tube. Two $\mu \mathrm{L}$ of 1:10 diluted DNA was used in each PCR reaction.

ARMS-PCR is a tetra-primer SNP genotyping strategy that combines two inner SNP-specific and two outer primers in a single reaction volume (7). Reactions are designed to produce up to three amplicons of diagnostic sizes which are then evaluated by agarose gel electrophoresis, as shown in Supplemental Figure S-1A. ARMS-PCR primer sets for ten SNPs across the Bbaa 2 region were developed using the recommended design program available online. (http:// cedar.genetics.soton.ac.uk/public html/ primer1.html). ARMS-PCR primer sequences are available in Supplemental Table S-1. PCR was performed in 96-well plates on a PTC-200 Thermal 
Table 1. PCR-Based SNP Genotyping Assay Comparison

\section{Estimated Reagent Cost and Throughput (per 96-well plate)}

ARMS-PCR Small Amplicon Blocked Probe

RFLP

Reagents (\$)

\begin{tabular}{|ccccc|}
\hline Taq / dNTP & 100 & 100 & 100 & 100 \\
\hline Primers / Probe & 2 & 1 & 2 & 1 \\
\hline Analysis & 2 & 10 & 10 & 22 \\
\hline Total Cost & 104 & 111 & 112 & 123
\end{tabular}

Time (Minutes)

\begin{tabular}{|ccccc|}
\hline PCR Setup & 30 & 30 & 30 & 30 \\
\hline Cycling & 90 & 75 & 75 & 90 \\
\hline Handling & 30 & 0 & 0 & 45 \\
Endonuclease & 0 & 0 & 0 & 60 \\
\hline Electrophoresis & 60 & 0 & 0 & 60 \\
\hline Analysis & 15 & 15 & 15 & 15 \\
\hline Total Time & 225 & 120 & 120 & 300 \\
\hline Bbaa2 Genotyping (26 positions, 96-well plates, run back-to-back) & \\
& ARMS-PCR & Small Amplicon Blocked Probe & RFLP
\end{tabular}

9 Unknowns

+3 Control

Samples

\begin{tabular}{|c|c|c|c|c|c|}
\hline & Total Cost $(\$)$ & 340 & 360 & 365 & 400 \\
\hline & Total Time (Hrs) & 15 & 8 & 8 & 20 \\
\hline \multicolumn{6}{|l|}{$\begin{array}{c}21 \text { Unknowns } \\
+3 \text { Control } \\
\text { Samples }\end{array}$} \\
\hline & Total Cost (\$) & 675 & 720 & 730 & 800 \\
\hline & Total Time (Hrs) & 27 & 14 & 14 & 35 \\
\hline \multicolumn{6}{|c|}{ Assay Design / Utility } \\
\hline & & ARMS-PCR & Small Amplicon & Blocked Probe & RFLP \\
\hline Success Rate & & $10 \%(1 / 10)$ & $81 \%(9 / 11)$ & $75 \%(12 / 16)$ & Not Tested \\
\hline Interpretation & & Unequivocal & Subjective* & Unequivocal & Unequivocal \\
\hline
\end{tabular}

Cycler using Platinum Taq (Invitrogen, Carlsbad, CA, USA) following the manufacturer's recommendations. PCR products were subjected to $1 \%$ agarose gel electrophoresis in 1X TBE buffer (89 $\mathrm{mM}$ Tris Base, $89 \mathrm{mM}$ Boric Acid, $2 \mathrm{mM}$ EDTA), and bands were visualized by ethidium bromide staining on a Gel Doc $\mathrm{XR}+$ platform (Bio-Rad Laboratories, Hercules, CA, USA).

Small Amplicon HRMA employs two primers closely surrounding the SNP of interest (8). Amplicon size is kept at a minimum, between 50-70 bp.
Next Generation Hamilton

\section{Automation Technology}

Your trusted partner in automated liquid handling

\section{A Pioneer in Liquid Handling Equipment}

Hamilton is the industry leader in the design and manufacture of liquid handling, process analytics, robotics and automated storage solutions. For more than 50 years, Hamilton has been satisfying customer needs by combining quality materials with skilled workmanship to ensure the highest level of performance. Hamilton takes pride in offering excellent service and support.

\section{NEW! Microlab ${ }^{\circledR}$ XRP}

Hamilton's most innovative liquid handler to hit the market, featuring:

Bring

organization to

the lab

$\checkmark$ Reduce lab

chaos while

increasing

productivity

Vertical

Integration

- Advanced Air

Pulse technology

- Advanced logistics

Innovative plate shuttling mechanism

\section{Microlab NIMBUS}

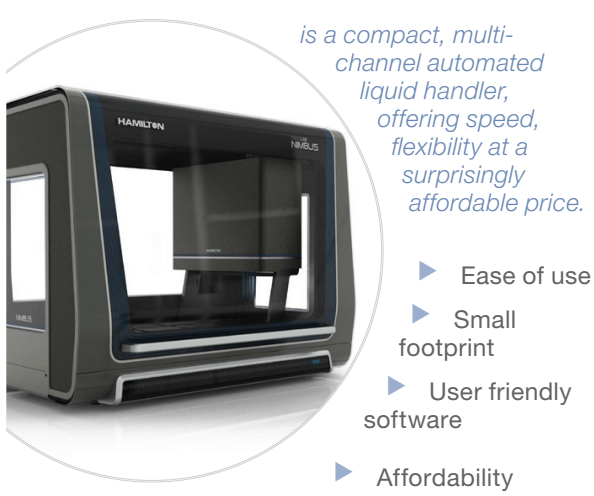

\section{Hamilton ensures customers}

Quality Service \& Application Support

Commitment to our Products

- Compliance (ISO 9001:2008, ISO 13485:2003 and 21 CFR 820)

Standard \& Custom Applications

\section{HAMILTON}

Contact Hamilton Today 
Table 2. Comparison of Discernable Genetic Landmarks in Bbaa2

\begin{tabular}{|c|c|c|c|}
\hline \multicolumn{2}{|c|}{ Bbaa2 Microsatellite Markers } & \multicolumn{2}{|c|}{ Bbaa2 SNP Genotyping Assays } \\
\hline bp & $\Delta$ bp & bp & $\Delta$ bp \\
\hline \multirow[t]{3}{*}{$119,175,776$} & N.A. & $119,172,092$ & N.A. \\
\hline & & $119,539,274$ & 367,182 \\
\hline & & $119,914,546$ & 375,272 \\
\hline \multirow[t]{7}{*}{$120,256,981$} & $1,081,205$ & $120,298,004$ & 383,458 \\
\hline & & $120,858,582$ & 560,578 \\
\hline & & $121,575,713$ & 717,131 \\
\hline & & $123,489,225$ & $1,913,512$ \\
\hline & & $123,905,461$ & 416,236 \\
\hline & & $124,668,171$ & 762,710 \\
\hline & & $125,034,265$ & 366,094 \\
\hline $125,309,605$ & $5,052,624$ & $125,316,596$ & 282,331 \\
\hline $125,581,535$ & 271,930 & $125,602,080$ & 285,484 \\
\hline \multirow[t]{2}{*}{$125,931,691$} & 350,156 & $125,940,137$ & 338,057 \\
\hline & & $126,607,294$ & 667,157 \\
\hline \multirow[t]{3}{*}{$127,404,260$} & $1,472,569$ & $127,391,403$ & 784,109 \\
\hline & & $128,208,712$ & 817,309 \\
\hline & & $128,954,717$ & 746,005 \\
\hline \multirow[t]{2}{*}{$129,042,314$} & $1,638,054$ & $129,338,863$ & 384,146 \\
\hline & & $130,476,763$ & $1,137,900$ \\
\hline \multirow[t]{3}{*}{$132,603,109$} & $3,560,795$ & $132,593,675$ & $2,116,912$ \\
\hline & & $133,538,654$ & 944,979 \\
\hline & & $134,737,636$ & $1,198,982$ \\
\hline $136,395,980$ & $3,792,871$ & $136,401,340$ & $1,663,704$ \\
\hline \multirow[t]{2}{*}{$137,534,245$} & $1,138,265$ & $137,497,474$ & $1,096,134$ \\
\hline & & $139,207,488$ & $1,710,014$ \\
\hline $141,162,992$ & $3,628,747$ & $141,158,294$ & $1,950,806$ \\
\hline Average & $2,198,722$ & Average & 879,448 \\
\hline Median & $1,555,312$ & Median & 746,005 \\
\hline StDev & $1,664,367$ & StDev & 576,607 \\
\hline $\operatorname{Max}$ & $5,052,624$ & $\operatorname{Max}$ & $2,116,912$ \\
\hline Min & 271,930 & Min & 282,331 \\
\hline
\end{tabular}

Distribution of previously used microsatellite markers and newly mapped SNP genotyping landmarks on mouse chromosome 5.

The hydrogen bonding characteristics of the nucleotide at the SNP position alters the melting temperature of the small amplicon, which can be detected by HRMA. The melting temperature differentials of homozygous Small Amplicons are reported to vary from 0.8 - 1.2 C, while heteroduplex amplicons are reported to melt at 2.0-3.0 C lower temperatures, as shown in Supplemental Figure S-1B.

Blocked (Unlabeled) probe HRMA employs a 3'phosporylated oligonucleotide probe that overlaps the SNP of interest but cannot be extended in the
PCR reaction, and two outer primers that produce a 65 - 150 bp amplicon (9). By including a limiting amount of primer on the same strand as the probe, asymmetric PCR produces an excess of opposite strand product to serve as a probe binding partner during HRMA. Probes form either perfect match or mismatch duplexes, with reported melting temperature differentials of $5.0-8.0 \mathrm{C}$, as shown in Supplemental Figure S-1C.

Small amplicon primer sets for 11 SNPs, and blocked probe primers and probes for 33 SNPs were developed using online Primer3 design software (http:// frodowi.mit.edu/primer3/). Small amplicon and blocked probe primer sets are available in Supplemental Tables S-2 and $S-3$, respectively. PCR and HRMA were performed in 96-well plates using Platinum Taq and 1x LCGreen Plus+ DNA intercalating dye (Idaho Technologies, Salt Lake City, UT, USA) on a LightCycler 480 platform (Roche Applied Science, Indianapolis, IN, USA). HRMA results were evaluated using LightCycler 480 Software Version 1.5.

LC480 Cycling Parameters were as follows: Initial Denaturation: $95^{\circ} \mathrm{C}(8$ min); Amplification: 60 cycles of $95^{\circ} \mathrm{C}$ denature $(4 \mathrm{~s}), 65^{\circ} \mathrm{C}$ annealing $(6 \mathrm{~s}), 72^{\circ} \mathrm{C}$ extension $(12 \mathrm{~s}) ;$ Melt: $95^{\circ} \mathrm{C}$ denature $(15 \mathrm{~s}), 50^{\circ} \mathrm{C}$ anneal $(60 \mathrm{~s}), 50-95^{\circ} \mathrm{C}$ continuous ramp at $0.06^{\circ} \mathrm{C} /$ second, 10 measurements per degree. Cooling: $50^{\circ} \mathrm{C}$ (10 s).

Each primer set was evaluated with $\mathrm{B} 6$, $\mathrm{C} 3 \mathrm{H}$, and 1:1 mixed $\mathrm{B} 6+\mathrm{C} 3 \mathrm{H}$ (obligate heterozygous) genomic DNA, as well as an $\mathrm{H}_{2} \mathrm{O}$ negative control.

Due to different assay design requirements, individual SNPs were not generally compatible with all three methodologies. SNPs were selected from the Perlegen 2 database (10). The Sanger Mouse Genomes Project database provides a useful additional resource to select or verify candidate SNPs for genotyping (6).

\section{Results and discussion}

The generation of congenic mouse strains is an iterative process. The ability to detect unique meiotic recombination events producing a novel isolated genomic interval in individual mice in a timely and cost effective manner is essential for efficient progress. By definition, an average of 25 out of 100 pups are expected to contain a unique crossover event within a $25 \mathrm{cM}$ locus. Therefore, as targeted intervals decrease in size, the number of expected new recombinants also decreases, leading to diminishing returns and increasing colony management costs. Theoretical calculations indicate that to narrow a single 25 $\mathrm{cM}$ locus to $5 \mathrm{cM}$ by breeding of ISCLs requires approximately 300 individuals, while further reducing each locus from 5 $\mathrm{cM}$ to $1 \mathrm{cM}$ requires approximately 380 additional individuals per regulatory gene (11). For the complex Bbaa2 locus, with multiple putative regulators within a $20+$ cM interval, this predicts that approximately 1820 individuals, the vast majority of which will be non-informative, will 
be required to achieve our goal of $1 \mathrm{cM}$ resolution for each putative locus.

Congenic breeders and their young pups can be maintained in a single cage, but are generally separated into 3 or more cages at weaning age. Since so many pups are non-informative as congenic intervals become narrow, rapid genotyping that allows efficient culling prior to weaning age may therefore provide a multiplier effect on cage cost savings, depending on the unique characteristics of individual colonies and institutional protocols. This process greatly benefits from the flexibility afforded by a high-throughput genotyping assay that can be rapidly and inexpensively performed on either small or large numbers of samples and can be targeted specifically to regions of interest within a sub-interval.

We previously relied on a panel of 11 microsatellite markers, some of which were poorly spaced or tightly clustered together, to genotype Bbaa 2 recombinants. To improve upon this, several methods of SNP genotyping were evaluated.

To ensure backward compatibility with previous microsatellite-based congenic genotyping data, SNP genotyping assays were first designed to approximate the positions of microsatellite markers in the Bbaa2 interval. Additional assays were then designed to further improve the distribution of landmarks across the interval.

All three tested SNP genotyping methodologies produced interpretable assays. However, ARMS-PCR (7) was not adopted for routine genotyping due to concerns about assay design and optimization, throughput, and the added risk of amplicon contamination during the obligatory handling and gel electrophoresis of PCR products. Another commonly used PCR-based SNP genotyping method, restriction fragment length polymorphism (RFLP) analysis, relies on incubation of the PCR amplicon with restriction endonucleases, followed by gel electrophoresis. These steps require additional reagents and equipment, and significantly increase assay turnaround time, which dramatically reduces throughput. Only a limited sub-set of SNPs are amenable to RFLP, and if RFLP-SNP mining is limited to commonly used, cost-effective restriction endonucleases that number is reduced even further. For the $22 \mathrm{Mb}$ Bbaa2 interval, only 538 such RFLP amenable SNPs were identified by SNP2RFLP (12) from over 19,000 high quality SNPs. Methodologically reducing the available SNPs in this way by more than 35 -fold may severely impair fine mapping of single genes or SNPs within specific congenic lines, especially in low-SNP areas of the genome. Like ARMS-PCR, these manipulations also require an open-tube assay that may increase the risk of amplicon contamination of laboratory space or equipment if samples are not handled appropriately.

Small amplicon HRMA (8) was found to be a reliable and efficient methodology. Nine out of 11 small amplicon SNP assays (81\%) produced interpretable results using standard reaction conditions. Heterozygous genotypes were readily apparent, due to the presence of a definitive heteroduplex melting peak. However, when scaled up to evaluate many unknown samples in parallel, inter-sample melting peak variability was observed, making it difficult to objectively assign a genotype to some individual homozygous samples. In all such cases, an objective genotype assignment could be made by repeating the HRMA analysis

\section{ANEW STANDARDIN RNA ISOLASION}

Just add water to the lysate to isolate RNA, with mRNA and small RNA (200-10 bases) isolated as separate fractions The single-step meth or without phase separation

No DNase treatment necessary

> RNA ready for RTPCR, microarrays and other applications

No need for reffigerated centrifuge

One reagent for solid and liquid samples
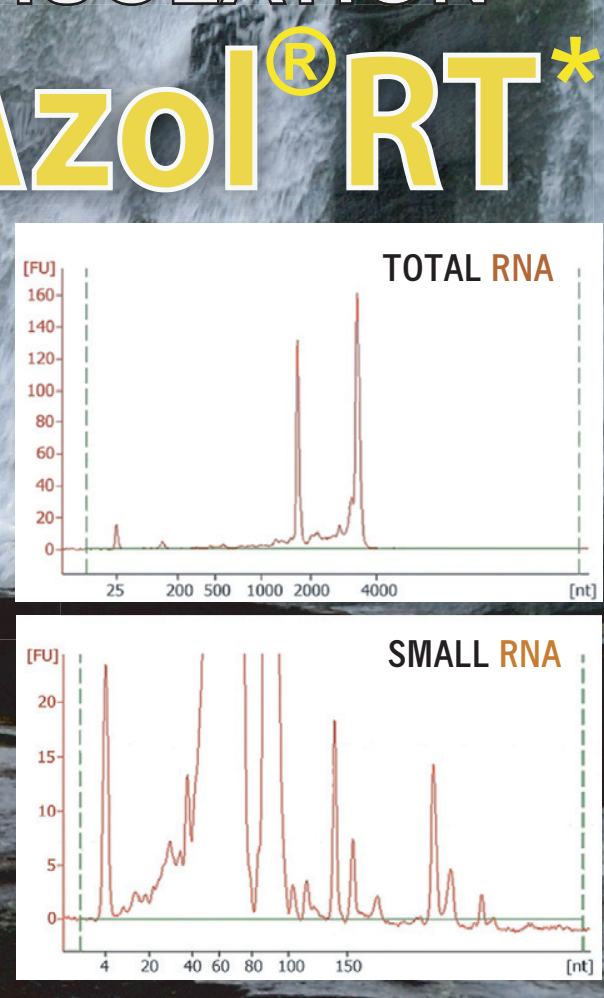
Table 3. Development of Advanced Bbaa2 Sub-Interval Congenic Lines

\begin{tabular}{|c|c|c|c|c|c|c|c|c|c|c|c|c|c|c|c|c|c|c|c|c|c|c|c|}
\hline & & & & & & & & & & Chr5 & 5 Positi & ion $(\mathrm{M}$ & & & & & & & & & & & \\
\hline 119.9 & 120.3 & 120.9 & 121.6 & 123.5 & 123.9 & 124.7 & 125.0 & 125.3 & 125.6 & 125.9 & 126.6 & 127.4 & 128.2 & 129.0 & 129.3 & 130.5 & 132.6 & 133.5 & 134.7 & 136.4 & 137.5 & 139.2 & 141.2 \\
\hline B & C & C & C & C & C & C & C & C & C & C & C & C & C & C & C & C & C & C & C & C & C & C & C \\
\hline B & C & C & C & C & C & C & C & C & C & C & C & C & C & C & C & C & C & C & C & C & B & B & B \\
\hline B & C & C & C & C & C & C & C & C & C & C & C & C & C & C & C & C & C & C & C & B & B & B & B \\
\hline B & C & C & C & C & C & C & C & C & C & C & C & C & C & C & C & C & B & B & B & B & B & B & B \\
\hline B & C & C & C & C & C & C & C & C & C & C & C & C & C & B & B & B & B & B & B & B & B & B & B \\
\hline B & C & C & C & C & C & C & C & C & C & C & C & B & B & B & B & B & B & B & B & B & B & B & B \\
\hline B & C & C & C & C & B & B & B & B & B & B & B & B & B & B & B & B & B & B & B & B & B & B & B \\
\hline B & C & C & C & B & B & B & B & B & B & B & B & B & B & B & B & B & B & B & B & B & B & B & B \\
\hline B & B & C & B & B & B & B & B & B & B & B & B & B & B & B & B & B & B & B & B & B & B & B & B \\
\hline B & B & B & B & B & B & B & B & B & B & B & B & B & B & C & C & C & C & C & C & C & C & C & C \\
\hline B & B & B & B & B & B & B & B & B & B & B & B & B & B & B & B & B & C & C & C & C & C & C & C \\
\hline B & B & B & B & B & B & B & B & B & B & B & B & B & B & B & B & B & B & B & C & C & C & C & C \\
\hline B & B & B & B & B & B & B & B & B & B & B & B & B & B & B & B & B & B & B & B & C & C & C & C \\
\hline \multirow{3}{*}{ B } & \multicolumn{23}{|c|}{$\mathrm{B} \mathrm{C} 3 \mathrm{~F}_{1} \times \mathrm{C} 3 \mathrm{H} / \mathrm{HeN} \mathrm{BC} 1$} \\
\hline & & & & & & & & & & & & & & & & & & & \multicolumn{5}{|c|}{$\mathrm{B} \mathrm{C} 3 \mathrm{~F}_{1}$ x C57BL/6 BC1 } \\
\hline & & & & & & & & & & & & & & & & & & & \multicolumn{5}{|c|}{ B6C3F $_{2}$ Intercross } \\
\hline
\end{tabular}

after spiking the unknown sample with known $\mathrm{B} 6$ or $\mathrm{C} 3 \mathrm{H}$ control DNA and looking for the presence of a heteroduplex melting peak.

Blocked probe HRMA (9) produced interpretable results under standard conditions in twelve out of sixteen assays (75\%) as originally designed. Even when evaluating many unknown samples in parallel, interpretation of working blocked probe sets was objective and unequivocal. Eleven out of 17 additional blocked probe assays designed across the region also produced interpretable assays, including three out of four designed as nearby replacements for non-working assays from the first round.

Both small amplicon and blocked probe HRMA were very rapid and efficient assays, generating SNP genotyping data in a 96-well format within 120 minutes. For very large scale applications, is unclear whether the observed difference in assay design success rates $(81 \%$ vs $75 \%)$ would strongly influence assay selection, or if other concerns would take precedence. Blocked probe HRMA offers the additional benefit over small amplicon HRMA of theoretically greater specificity due to the addition of a third sequence-specific oligonucleotide as a probe, as well as dramatically better separation between diagnostic peaks so that spiking and HRMA repetition was not necessary. For these reasons we have adopted blocked probe HRMA as our preferred methodology for highthroughput SNP genotyping.

The estimated time, reagent cost, and utility of the ARMS-PCR, small 
amplicon, blocked probe, and RFLP methods based on uniform manufacturer recommended $50 \mu \mathrm{L}$ PCR cycling volumes are compared in Table 1 .

All Bbaa2 HRMA genotyping assays have been successfully scaled down to $10 \mu \mathrm{L}$ reaction volumes in 96 -well plates while retaining diagnostic integrity, further reducing reagent costs. Rapid turnaround times have enabled complete genotyping of the Bbaa2 interval or appropriate sub-interval in all congenic offspring prior to weaning age, also reducing cage costs. This has allowed resources to be better focused on expanding breeding cages to accelerate the generation of new recombinants and on maintenance of novel sub-interval congenic lines.

The use of SNP genotyping by HRMA is becoming increasingly accessible as equipment capable of performing HRMA becomes more common in research institutions around the world. A search of the PubMed database in October 2011 identified over 240 publications using high resolution melting in the past year alone, primarily relating to human diagnostics. For this purpose, the SNPs of interest are pre-defined due to their estab- lished linkage to or causation of human disease. While we have found that a large majority of SNPs can be genotyped in this way, not every such SNP is amenable to HRMA genotyping, depending upon the unique characteristics and complexity of surrounding DNA sequence. However, for the purposes of mapping inbred congenic mouse strains, the genomic location of a SNP rather than its potential biological function is the primary consideration, allowing flexibility for several different SNPs to be evaluated in a given area. Based on the Perlegen high-density resequencing project, SNPs differing between any two pairwise combinations of 12 classic inbred strains occur with an average frequency ranging from 1 in $440 \mathrm{bp}$ to 1 in $21,000 \mathrm{bp}$ in high-SNP or low-SNP regions of the mouse genome, respectively (13). Even in low frequency regions, this represents a potential resolution for congenic mapping on the order of $0.01-0.10 \mathrm{cM}$.

The Bbaa2 region of mouse chromosome 5 is complex. It exhibits a nonlinear relationship between basepair position and centiMorgan distance (Figure 1A), suggesting that meiotic recombination does not occur with uniform frequency across the interval. It also exhibits a highly variable SNP density, with some areas highly conserved between our parental B6 and $\mathrm{C} 3 \mathrm{H}$ inbred strains of interest (Figure 1B). These characteristics impact the capacity to generate congenic animals and then to identify where recombination has occurred. Despite this complexity, diagnostic SNP genotyping assays were successfully designed at regular intervals. A total of 26 SNP genotyping assays across the Bbaa2 region are now used for routine genotyping of backcross progeny in our congenic colony. By adopting SNP genotyping methods, all previously used microsatellite markers have been replaced with approximately equivalent high-throughput SNP assays, and 15 additional landmarks have been added. As shown in Table 2, the average and median resolutions between genotyping landmarks across the Bbaa2 interval have improved from $2.20 \mathrm{Mbp}$ and $1.56 \mathrm{Mbp}$ to $0.88 \mathrm{Mbp}$ and 0.75 Mbp, respectively. The ability to readily design new genotyping assays targeted to intervals containing novel breakpoints in individual mice contributes to a harmonious and efficient refinement

\section{Drummond

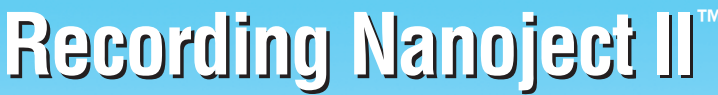

\section{Enables High Precision Electrophysiological Guidance of Nanoliter Injections into the Brain}

- Facilitates connection to an extracellular amplifier to allow recording of neural activity

- Supplements stereotaxic coordinates in guiding the placement of small injections

- Provides direct displacement of neuronal tracer or excitotoxin from the pipet

The Drummond Recording Nanoject II was specifically developed to improve micro-volume injection techniques used to study connectivity and function of specific brain nuclei. Designed to be operated in conjunction with an extracellular amplifier, the integrated system can be used to reliably place small volumes into targeted areas. The Recording Nanoject II is engineered to easily mount on popular stereotaxic instruments.

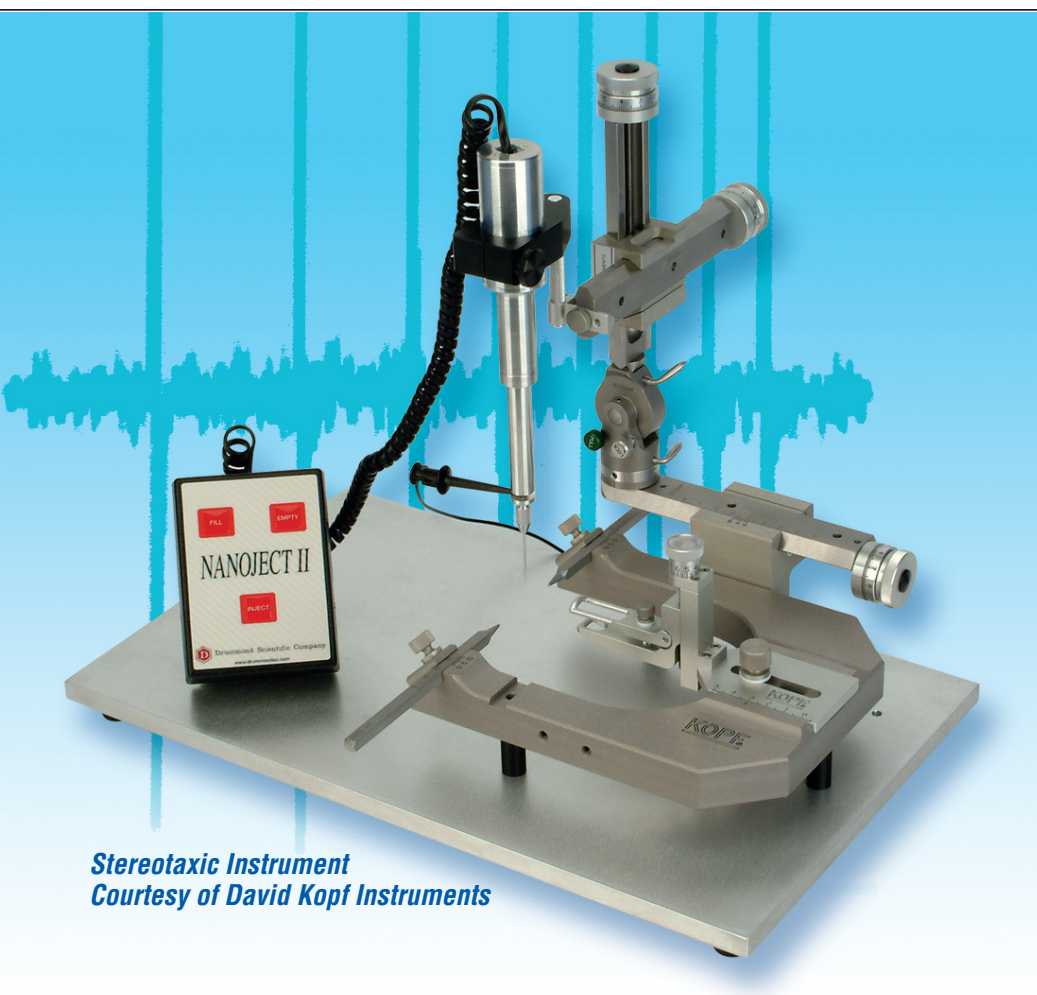

DRUMMOND SCIENTIFIC COMPANY

500 Parkway, Box 700

Broomall, PA 19008

1-800/523-7480 • FAX: 1-610/353-6204

Web site: www.drummondsci.com 
process. In many cases, the prevalence of discernable SNPs will provide great enough resolution to exclude single genes, or even single SNPs within genes, from a congenic interval. This is especially important when attempting to precisely define the boundaries of similar or overlapping recombinant congenic lines. The use of HRMA based SNP genotyping may also be of special interest to anyone working with recombinant inbred (RI) lines such as those produced by the Collaborative Cross (14), or performing iterative backcrossing, such as during the transfer of a targeted gene knockout to a specific genetic background. A basic SNP genotyping panel for a broad interval surrounding the gene of interest may help speed up the backcrossing process by quickly identifying which individuals within litters inherited the narrowest surrounding interval, and would therefore best serve as breeders for successive backcrosses.

Our adoption of HRMA-based SNP genotyping has facilitated the generation, identification, and more precise discernment of 20 novel ISCLs carrying unique subintervals of the Bbaa2 locus, as shown in Table 3. Many of these newly defined advanced congenics coincide with regions previously predicted to encode loci with positive and negative effects on Lyme arthritis severity, thereby establishing tools for the formal analysis of regulatory regions on chromosome 5. Current congenic lines allow the pairwise interrogation of intervals as small as approximately $0.6 \mathrm{Mbp}$, which will greatly facilitate efforts to identify causal genes in the region, and may be of general interest for the investigation of any phenotypic differences assigned to this region of mouse chromosome 5.

\section{Acknowledgments}

We would like to acknowledge the helpful advice and expertise provided by Dr. Carl Wittwer and Luming Zhou during our implementation of High Resolution Melting Analysis assays. Heydon Kaddas provided helpful assistance with the design and testing of Bbaa2 SNP genotyping primer sets. The project described was supported by Award Number T32AI055434 (KCB), AI-24158 \& AI-088451(JHW), AI32223 (JJW) from the National Institute Of Allergy And Infectious Diseases and AR-43521 (CT \& JJW) from the National Institute of Arthritis and Musculoskeletal and Skin Diseases. The DNA/Peptide Core is supported in part by the Cancer Center Support Grant P30 CA04201. The content is solely the responsibility of the authors and does not necessarily represent the official views of the National Institutes of Health. This paper is subject to the NIH Public Access Policy.

\section{Competing interests}

The authors declare no competing interests.

\section{References}

1. Crick, F. 1970. Central dogma of molecular biology. Nature 227:561-563.

2. Weis, J.J., B.A. McCracken, Y. Ma, D. Fairbairn, R.J. Roper, T.B. Morrison, J.H. Weis, J.F. Zachary, et al. 1999. Identification of quantitative trait loci governing arthritis severity and humoral responses in the murine model of Lyme disease. J. Immunol. 162:948-956.

3. Roper, R.J., J.J. Weis, B.A. McCracken, C.B. Green, Y. Ma, K.S. Weber, D. Fairbairn, R.J. Butterfield, et al. 2001. Genetic control of susceptibility to experimental Lyme

\section{amsbio CELLBANKER cryopreservation}

- 20 years of cell freezing experience

- Avoid controlled freezing \& liquid nitrogen

- High cell viability for cell lines, primary \& stem cells

- Serum free/ Pharmacopeia chemically defined arthritis is polygenic and exhibits consistent linkage to multiple loci on chromosome 5 in four independent mouse crosses. Genes Immun. 2:388-397.

4. Ma, Y., J.C. Miller, H. Crandall, E.T. Larsen, D.M. Dunn, R.B. Weiss, M. Subramanian, J.H. Weis, et al. 2009. Interval-specific congenic lines reveal quantitative trait Loci with penetrant lyme arthritis phenotypes on chromosomes 5, 11, and 12. Infect. Immun. 77:3302-3311.

5. Smigielski, E.M., K. Sirotkin, M. Ward, and S.T. Sherry. 2000. dbSNP: a database of single nucleotide polymorphisms. Nucleic Acids Res. 28:352-355.

6. Keane, T.M., L. Goodstadt, P. Danecek, M.A. White, K. Wong, B. Yalcin, A. Heger, A. Agam, et al. 2011. Mouse genomic variation and its effect on phenotypes and gene regulation. Nature 477:289-294.

7. Ye, S., S. Dhillon, X. Ke, A.R. Collins, and I.N. Day. 2001. An efficient procedure for genotyping single nucleotide polymorphisms. Nucleic Acids Res. 29:E88-8.

8. Liew, M., R. Pryor, R. Palais, C. Meadows, M. Erali, E. Lyon, and C. Wittwer. 2004 Genotyping of single-nucleotide polymorphisms by high-resolution melting of small amplicons. Clin. Chem. 50:1156-1164.

9. Zhou, L., A.N. Myers, J.G. Vandersteen, L. Wang, and C.T. Wittwer. 2004. Closed-tube genotyping with unlabeled oligonucleotide probes and a saturating DNA dye. Clin. Chem. 50:1328-1335.

10. Cox, D. and K.A. Frazer. (2011) SNP data, $8.2+$ million locations for 16 inbred strains of mice. MPD:Perlegen2. Mouse Phenome Database web site, The Jackson Laboratory, Bar Harbor, Maine USA. http://phenome. jax.org

11. Darvasi, A. 1998. Experimental strategies for the genetic dissection of complex traits in animal models. Nat. Genet. 18:19-24.

12. Beckstead, W.A., B.C. Bjork, R.W. Stottmann, S. Sunyaev, and D.R. Beier. 2008. SNP2RFLP: a computational tool to facilitate genetic mapping using benchtop analysis of SNPs. Mamm. Genome 19:687-690.

13. Frazer, K.A., E. Eskin, H.M. Kang, M.A. Bogue, D.A. Hinds, E.J. Beilharz, R.V. Gupta, J. Montgomery, et al. 2007. A sequence-based variation map of 8.27 million SNPs in inbred mouse strains. Nature 448:1050-1053.

14. Churchill, G.A., D.C. Airey, H. Allayee, J.M. Angel, A.D. Attie, J. Beatty, W.D. Beavis, J.K. Belknap, et al. 2004. The Collaborative Cross, a community resource for the genetic analysis of complex traits. Nat. Genet. 36:1133-1137.

Received 31 October 2011; accepted 13 January 2012.

Address correspondence to Janis J. Weis, Department of Pathology, University of Utah, Salt Lake City, UT, USA. Email: janis.weis@ path.utah.edu

To purchase reprints of this article, contact: biotechniques@fosterprinting.com 